\title{
Symmetry of Positive Solutions to Quasilinear Fractional Systems
}

\section{Phuong Le}

Abstract. Using the method of moving planes, we establish the radial symmetry of positive solutions to the fractional system

$$
\left\{\begin{array}{l}
(-\Delta)_{p}^{s} u=f(u, v), \\
(-\Delta)_{q}^{t} v=g(u, v)
\end{array}\right.
$$

in the entire Euclidean space $\mathbb{R}^{n}$ and in the unit ball, where $0<s, t<1$ and $p, q \geq$ 2. In particular, our result can be applied to the nonlinearities $f(u, v) \equiv u^{a} v^{b}$ and $g(u, v) \equiv u^{c} v^{d}$, where $a, d \in \mathbb{R}$ and $b, c>0$.

\section{Introduction}

This paper is concerned with the symmetry of positive solutions to the quasilinear fractional systems

$$
\begin{cases}(-\Delta)_{p}^{s} u=f(u, v) & \text { in } B_{1}, \\ (-\Delta)_{q}^{t} v=g(u, v) & \text { in } B_{1}, \\ u=v=0 & \text { in } \mathbb{R}^{n} \backslash B_{1}\end{cases}
$$

and

$$
\begin{cases}(-\Delta)_{p}^{s} u=f(u, v) & \text { in } \mathbb{R}^{n} \\ (-\Delta)_{q}^{t} v=g(u, v) & \text { in } \mathbb{R}^{n}\end{cases}
$$

where $B_{1}$ denotes the unit ball in $\mathbb{R}^{n}, 0<s, t<1$ and $p, q \geq 2$.

The fractional $p$-Laplacian $(-\Delta)_{p}^{s}$, which appears in (1.1) and (1.2), is a nonlinear nonlocal operator. It is defined as

$$
\begin{aligned}
(-\Delta)_{p}^{s} u(x) & =C_{n, s, p} \mathrm{PV} \int_{\mathbb{R}^{n}} \frac{|u(x)-u(y)|^{p-2}[u(x)-u(y)]}{|x-y|^{n+s p}} d y \\
& =C_{n, s, p} \lim _{\varepsilon \rightarrow 0^{+}} \int_{\mathbb{R}^{n} \backslash B_{\varepsilon}(x)} \frac{|u(x)-u(y)|^{p-2}[u(x)-u(y)]}{|x-y|^{n+s p}} d y
\end{aligned}
$$

Received April 24, 2020; Accepted December 3, 2020.

Communicated by Jann-Long Chern.

2020 Mathematics Subject Classification. 35R11, 35J92, 35B06.

Key words and phrases. quasilinear fractional system, fractional $p$-Laplacian, symmetry of solutions. 
where $\mathrm{PV}$ is an abbreviation of the Cauchy principal value, $C_{n, s, p}$ is a normalization constant and $B_{\varepsilon}(x)$ denotes the ball of center $x \in \mathbb{R}^{n}$ and radius $\varepsilon>0$. Clearly, $(-\Delta)_{p}^{s} u(x)$ is well-defined for $u \in L_{s, p} \cap C_{\text {loc }}^{1,1}(\Omega)$ and $x \in \Omega$, where $\Omega \subset \mathbb{R}^{n}$ is an open domain and

$$
L_{s, p}=\left\{u \in L_{\mathrm{loc}}^{p-1}\left(\mathbb{R}^{n}\right) \mid \int_{\mathbb{R}^{n}} \frac{|1+u(x)|^{p-1}}{1+|x|^{n+s p}} d x<\infty\right\},
$$

see 9,24 for more details. The operator $(-\Delta)_{q}^{t}$ can be defined in the same way.

The fractional $p$-Laplacian arises in some important applications such as the nonlocal "Tug-of-War" game [3,4]. It is also a generalization of the well-known fractional Laplacian, which has been widely used to model various physical phenomena, such as turbulence, water waves, anomalous diffusion, phase transitions, flame propagation and quasi-geostrophic flows, see [5,7, 14]. The fractional Laplacian also appears in probability, optimization and finance, see [1,2,6].

In order to study the symmetry properties of solutions to equations involving the fractional Laplacian, one may exploit some notable methods such as the extension method by Caffarelli and Silvestre [6], the integral equation method by Chen, Li and Ou [11] and the direct method of moving planes by Chen, Li and Li 10] (see also [15,16]). Several works in literature follow these methods to establish symmetry, monotonicity and nonexistence results for various types of systems involving the fractional Laplacian, see $13,17,18,20$, $21,23,25,27,28$ and the references therein.

However, due to its degeneracy, the fractional $p$-Laplacian cannot be studied by the above methods. Recently, Chen and Li [9] introduced a variant of the direct moving plane method. Using this new tool, they are able to establish the symmetry of positive solutions to some fractional $p$-Laplacian equations in $\mathbb{R}^{n}$ and $B_{1}$.

More precisely, in [9, Theorem 4], Chen and Li showed that, if $u$ is a positive solution of the equation

$$
\begin{cases}(-\Delta)_{p}^{s} u=f(u) & \text { in } B_{1}, \\ u=0 & \text { in } \mathbb{R}^{n} \backslash B_{1},\end{cases}
$$

where $f(u) \equiv u^{q}$ with $q \geq p-1$, then $u$ must be radially symmetric and monotone decreasing about the origin. Later, Wu and Niu [26] extend the result to any Lipschitz continuous function $f$ (see [26, Theorem 1.2]). A symmetry result for system (1.1) was recently established in 8 under some technical assumptions on $f$ and $g$. One of our purposes in writing this paper is to improve [8] by establishing the symmetry of positive solutions to (1.1) for a very large class of $f$ and $g$. Our first result reads as follows.

Theorem 1.1. Let $f, g \in C_{\mathrm{loc}}^{0,1}([0, \infty) \times[0, \infty))$ be such that $f(z, \cdot)$ and $g(\cdot, z)$ are nondecreasing in $(0, \infty)$ for all $z>0$. Assume that $(u, v) \in C^{1,1}\left(\bar{B}_{1}\right) \times C^{1,1}\left(\bar{B}_{1}\right)$ is a positive solution of (1.1). Then $u$ and $v$ must be radially symmetric and monotone decreasing about the origin. 
The second objective of the present paper is to establish the symmetry of positive solutions to system (1.2). In [9, Theorem 5], Chen and Li studied the equation

$$
(-\Delta)_{p}^{s} u=f(u) \quad \text { in } \mathbb{R}^{n}
$$

They showed that, if $f^{\prime}(z) \leq 0$ for $z$ sufficiently small and $u$ is a decaying positive solution, then $u$ must be radially symmetric and monotone decreasing about some point. Notice that an analogous result for system $(1.2)$ was currently obtained by Chen and Liu in [12]. They assumed $f, g \in C^{1}([0, \infty) \times[0, \infty))$ be such that

(i) $f_{v}^{\prime}(u, v)>0, g_{u}^{\prime}(u, v)>0$ for all $u, v>0$,

(ii) $f_{u}^{\prime}(0,0)<0, g_{v}^{\prime}(0,0)<0, f_{u}^{\prime}(0,0) g_{v}^{\prime}(0,0)-f_{v}^{\prime}(0,0) g_{u}^{\prime}(0,0)>0$.

Then they proved that $u$ and $v$ must be radially symmetric and monotone decreasing about the same point if $(u, v)$ is a decaying positive solution to $[1.2)$ (see [12, Theorem 1]). One may observe that, because of the restriction (ii), Chen and Liu's result cannot be applied to the fractional Schrödinger system

$$
\begin{cases}(-\Delta)_{p}^{s} u=u^{a} v^{b} & \text { in } \mathbb{R}^{n} \\ (-\Delta)_{q}^{t} v=u^{c} v^{d} & \text { in } \mathbb{R}^{n} .\end{cases}
$$

One may ask whether we can obtain the symmetry of some kind of decaying positive solutions to system (1.4) with $a, b, c, d>0$, or more generally, to system 1.2 without the conditions $f_{u}^{\prime} \leq 0$ and $g_{v}^{\prime} \leq 0$ for $(u, v) \approx(0,0)$. We give a positive answer to this question in the following theorem.

Theorem 1.2. Let $\alpha, \beta>0$ and $f, g \in C^{1}((0, \infty) \times(0, \infty))$ be such that

(i) $f_{v}^{\prime}(u, v)>0, g_{u}^{\prime}(u, v)>0$ for all $u, v>0$,

(ii) $f_{u}^{\prime}(u, v) \leq 0$ or $f_{u}^{\prime}(u, v)=o\left(u^{a_{1}} v^{b_{1}}\right)$ as $(u, v) \rightarrow\left(0^{+}, 0^{+}\right)$, where $a_{1}, b_{1} \geq 0$ satisfy $\alpha a_{1}+\beta b_{1} \geq \alpha(p-2)+s p$,

(iii) $f_{v}^{\prime}(u, v)=o\left(u^{a_{2}} v^{b_{2}}\right)$ as $(u, v) \rightarrow\left(0^{+}, 0^{+}\right)$, where $a_{2}, b_{2} \geq 0$ satisfy $\alpha a_{2}+\beta b_{2} \geq$ $\alpha(p-2)+s p$,

(iv) $g_{u}^{\prime}(u, v)=o\left(u^{c_{1}} v^{d_{1}}\right)$ as $(u, v) \rightarrow\left(0^{+}, 0^{+}\right)$, where $c_{1}, d_{1} \geq 0$ satisfy $\alpha c_{1}+\beta d_{1} \geq$ $\beta(q-2)+t q$,

(v) $g_{v}^{\prime}(u, v) \leq 0$ or $g_{v}^{\prime}(u, v)=o\left(u^{c_{2}} v^{d_{2}}\right)$ as $(u, v) \rightarrow\left(0^{+}, 0^{+}\right)$, where $c_{2}, d_{2} \geq 0$ satisfy $\alpha c_{2}+\beta d_{2} \geq \beta(q-2)+t q$. 
Assume that $(u, v) \in\left(L_{s, p} \cap C_{\mathrm{loc}}^{1,1}\left(\mathbb{R}^{n}\right)\right) \times\left(L_{t, q} \cap C_{\mathrm{loc}}^{1,1}\left(\mathbb{R}^{n}\right)\right)$ is a positive solution of 1.2 ) such that

$$
u(x) \sim \frac{1}{|x|^{\alpha}} \quad \text { and } \quad v(x) \sim \frac{1}{|x|^{\beta}} \quad \text { as }|x| \rightarrow \infty .
$$

Then $u$ and $v$ must be radially symmetric and monotone decreasing about the same point.

Remark 1.3. Since the Kelvin transform is no longer valid for the fractional $p$-Laplacian, we need to impose the additional assumptions on the behavior of $u$ and $v$ at infinity. This is the same for other works involving the symmetry results for fractional $p$-Laplacian in the literature to the best of our knowledge. Theorem 1.2 can be seen as an extension of Theorems 1 and 4 in 21 to the quasilinear case $p, q \geq 2$.

Theorem 1.2 can be applied to system $(1.4)$ with $a, d \in(-\infty, 0] \cup[1,+\infty)$ and $b, c \in$ $[1,+\infty)$ but cannot deal with the case $a, b, c$ or $d \in(0,1)$. However, by conducting a finer analysis, we are able to cover also the case $a, b, c, d \in(0,1)$ for system (1.4) as follows.

Theorem 1.4. Let $\alpha, \beta>0$ and $a, b, c, d \in \mathbb{R}$ be such that

(i) $a \leq 0$, or $a>0$ and $\alpha(a-1)+\beta b>\alpha(p-2)+s p$,

(ii) $b>0$ and $\alpha a+\beta(b-1)>\alpha(p-2)+s p$,

(iii) $c>0$ and $\alpha(c-1)+\beta d>\beta(q-2)+t q$,

(iv) $d \leq 0$, or $d>0$ and $\alpha c+\beta(d-1)>\beta(q-2)+t q$.

Assume that $(u, v) \in\left(L_{s, p} \cap C_{\mathrm{loc}}^{1,1}\left(\mathbb{R}^{n}\right)\right) \times\left(L_{t, q} \cap C_{\mathrm{loc}}^{1,1}\left(\mathbb{R}^{n}\right)\right)$ is a positive solution of (1.4) such that

$$
u(x) \sim \frac{1}{|x|^{\alpha}} \quad \text { and } \quad v(x) \sim \frac{1}{|x|^{\beta}} \quad \text { as }|x| \rightarrow \infty .
$$

Then $u$ and $v$ must be radially symmetric and monotone decreasing about the same point.

In particular, when $a=r-1, b=1, c=r, d=0$ and $q=2$, Theorem 1.4 reduces to a known result by Ma and Zhang [22], which involves the symmetry of positive solutions to the static Choquard type equation

$$
(-\Delta)_{p}^{s} u=\left(\frac{1}{|x|^{n-2 t}} * u^{r}\right) u^{r-1} \quad \text { in } \mathbb{R}^{n} .
$$

More precisely, Ma and Zhang transformed (1.5) to the fractional system

$$
\begin{cases}(-\Delta)_{p}^{s} u=u^{r-1} v & \text { in } \mathbb{R}^{n}, \\ (-\Delta)^{t} v=u^{r} & \text { in } \mathbb{R}^{n} .\end{cases}
$$

Then they proved the following result, which is exactly a corollary of Theorem 1.4 . 
Corollary 1.5. 22, Theorem 1.1] Let $r>p-1$ be such that

$$
\alpha>\max \left\{\frac{s p}{r-p+1}, \frac{2 t}{r-1}\right\} \quad \text { and } \quad \alpha(r-p)+\beta>s p .
$$

Assume that $(u, v) \in\left(L_{s, p} \cap C_{\mathrm{loc}}^{1,1}\left(\mathbb{R}^{n}\right)\right) \times\left(L_{t, 2} \cap C_{\mathrm{loc}}^{1,1}\left(\mathbb{R}^{n}\right)\right)$ is a positive solution of 1.6 such that

$$
u(x) \sim \frac{1}{|x|^{\alpha}} \quad \text { and } \quad v(x) \sim \frac{1}{|x|^{\beta}} \quad \text { as }|x| \rightarrow \infty .
$$

Then $u$ and $v$ must be radially symmetric and monotone decreasing about the same point.

Notice that a better symmetry result for 1.5 was obtained recently by the author in [19 using more direct analysis.

Finally, we may state a corollary of Theorem 1.2 for $(1.3)$ as follows.

Corollary 1.6. Let $f \in C^{1}((0, \infty))$ be such that

$$
f^{\prime}(z)=O\left(z^{a-1}\right) \quad \text { as } z \rightarrow 0^{+}
$$

where $a>p-1$. Assume that $u \in L_{s, p} \cap C_{\mathrm{loc}}^{1,1}\left(\mathbb{R}^{n}\right)$ is a positive solution of (1.3) such that

$$
u(x) \sim \frac{1}{|x|^{\alpha}} \quad \text { as }|x| \rightarrow \infty
$$

with $\alpha>\frac{s p}{a-p+1}$. Then $u$ must be radially symmetric and monotone decreasing about some point.

Remark 1.7. To see Corollary 1.6 follows from Theorem 1.2 , one just notice that, if $u$ is a positive solution of $(1.3)$, then $(u, u)$ is a positive solution of the system

$$
\begin{cases}(-\Delta)_{p}^{s} u=f(u)-u^{a}+v^{a} & \text { in } \mathbb{R}^{n}, \\ (-\Delta)_{p}^{s} v=f(v)-v^{a}+u^{a} & \text { in } \mathbb{R}^{n} .\end{cases}
$$

Remark 1.8. Corollary 1.6 has been proved in $[9$, Theorem 6$]$ for the special case $f(z) \equiv z^{a}$ with $a>p-1$. Here we only assume that $f^{\prime}(z)$ is bounded by $C z^{a-1}$ for sufficiently small $z$.

The remainder of the present paper is organized as follows. In Section 2, we introduce some notations and lemmas which will be used later. In Section 3 , we prove Theorem 1.1 . In Section 4, we derive a decay at infinity principle and combine it with the moving plane method to prove Theorems 1.2 and 1.4 .

For brevity, we use $C$ to denote various positive constants which may varies from place to place. In some situations, we append subscripts to $C$ to specify its dependence on the subscript parameters. We also denote $B_{\varepsilon}=B_{\varepsilon}(0), z^{+}=\max \{z, 0\}$ and $z^{-}=\min \{z, 0\}$ for any $\varepsilon>0$ and $z \in \mathbb{R}$. 


\section{Preliminaries}

Throughout the paper, we use the following notations to facilitate our description. For arbitrary $\lambda \in \mathbb{R}$, let

- $T_{\lambda}=\left\{x \in \mathbb{R}^{n} \mid x_{1}=\lambda\right\}$ be the moving plane,

- $\Sigma_{\lambda}=\left\{x \in \mathbb{R}^{n} \mid x_{1}<\lambda\right\}$ be the left region of the plane,

- $x^{\lambda}=\left(2 \lambda-x_{1}, x_{2}, \ldots, x_{n}\right)$ be the reflection of the point $x=\left(x_{1}, x_{2}, \ldots, x_{n}\right)$ about the plane $T_{\lambda}$.

We also define $u_{\lambda}(x)=u\left(x^{\lambda}\right), v_{\lambda}(x)=v\left(x^{\lambda}\right)$ and introduce functions

$$
U_{\lambda}(x)=u_{\lambda}(x)-u(x), \quad V_{\lambda}(x)=v_{\lambda}(x)-v(x) .
$$

One important ingredient of the moving plane method for the fractional $p$-Laplacian is the following lemma.

Lemma 2.1 (A boundary estimate 9 ). Assume that $U_{\lambda_{0}} \geq 0$ in $\Sigma_{\lambda_{0}}$ and $U_{\lambda_{0}} \not \equiv 0$ in $\Sigma_{\lambda_{0}}$. Suppose $\lambda_{k} \searrow \lambda_{0}$ and $x^{k} \in \Sigma_{\lambda_{k}}$ such that

$$
U_{\lambda_{k}}\left(x^{k}\right)=\min _{\Sigma_{\lambda_{k}}} U_{\lambda_{k}} \leq 0 \quad \text { and } \quad x^{k} \rightarrow x^{0} \in T_{\lambda_{0}} .
$$

Let $\delta_{k}=\operatorname{dist}\left(x^{k}, T_{\lambda_{k}}\right)=\lambda_{k}-x_{1}^{k}$. Then

$$
\limsup _{\delta_{k} \rightarrow 0} \frac{1}{\delta_{k}}\left[(-\Delta)_{p}^{s} u_{\lambda_{k}}\left(x^{k}\right)-(-\Delta)_{p}^{s} u\left(x^{k}\right)\right]<0 .
$$

Remark 2.2. In the original statement (see [9, Theorem 2.3]), $U_{\lambda_{0}}$ is assumed to be positive in all of $\Sigma_{\lambda_{0}}$. However, it is clear from its proof in [9] that this assumption can be weaken as $U_{\lambda_{0}} \geq 0$ in $\Sigma_{\lambda_{0}}$ and $U_{\lambda_{0}} \not \equiv 0$ in $\Sigma_{\lambda_{0}}$, like we stated above. In applications, however, one usually need $U_{\lambda_{0}}>0$ to guarantee that the limit of $\left\{x^{k}\right\}$ belongs to $T_{\lambda_{0}}$.

The following elementary inequality was also proved in $[9$.

Lemma 2.3. 9, Lemma 5.1] Let $G(z)=|z|^{p-2} z$, then $G$ is strictly increasing in $\mathbb{R}$ and

$$
G(w)-G(z) \leq C|z|^{p-2}(w-z)
$$

for all $w \leq z$.

To deal with problems in $\mathbb{R}^{n}$, we need the following estimation. 
Lemma 2.4. Let $u \in L_{s, p} \cap C_{\mathrm{loc}}^{1,1}\left(\mathbb{R}^{n}\right)$ be a positive function such that

$$
u(x) \sim \frac{1}{|x|^{\alpha}} \quad \text { as }|x| \rightarrow \infty
$$

for some $\alpha>0$. Assume $\lambda<\bar{\lambda}<0$ and $U_{\lambda}\left(x^{*}\right)=\min _{\Sigma_{\lambda}} U_{\lambda} \leq 0$ for some $x^{*} \in \Sigma_{\lambda}$. Then we have

$$
(-\Delta)_{p}^{s} u_{\lambda}\left(x^{*}\right)-(-\Delta)_{p}^{s} u\left(x^{*}\right) \leq \frac{C U_{\lambda}\left(x^{*}\right)}{\left|x^{*}\right|^{\alpha(p-2)+s p}}
$$

where $C$ depends on $\bar{\lambda}$, but is independent of $\lambda$.

Proof. Denoting $G(z)=|z|^{p-2} z$ and using the definition of fractional $p$-Laplacian, we can compute

$$
\begin{aligned}
& (-\Delta)_{p}^{s} u_{\lambda}\left(x^{*}\right)-(-\Delta)_{p}^{s} u\left(x^{*}\right) \\
& =C_{n, s, p} \mathrm{PV} \int_{\mathbb{R}^{n}} \frac{G\left(u_{\lambda}\left(x^{*}\right)-u_{\lambda}(y)\right)-G\left(u\left(x^{*}\right)-u(y)\right)}{\left|x^{*}-y\right|^{n+s p}} d y \\
& =C_{n, s, p} \mathrm{PV} \int_{\Sigma_{\lambda}} \frac{G\left(u_{\lambda}\left(x^{*}\right)-u_{\lambda}(y)\right)-G\left(u\left(x^{*}\right)-u(y)\right)}{\left|x^{*}-y\right|^{n+s p}} d y \\
& +C_{n, s, p} \int_{\Sigma_{\lambda}} \frac{G\left(u_{\lambda}\left(x^{*}\right)-u(y)\right)-G\left(u\left(x^{*}\right)-u_{\lambda}(y)\right)}{\left|x^{*}-y^{\lambda}\right|^{n+s p}} d y \\
& =C_{n, s, p} \mathrm{PV} \int_{\Sigma_{\lambda}}\left(\frac{1}{\left|x^{*}-y\right|^{n+s p}}-\frac{1}{\left|x^{*}-y^{\lambda}\right|^{n+s p}}\right) \\
& \times\left[G\left(u_{\lambda}\left(x^{*}\right)-u_{\lambda}(y)\right)-G\left(u\left(x^{*}\right)-u(y)\right)\right] d y \\
& +C_{n, s, p} \int_{\Sigma_{\lambda}} \frac{G\left(u_{\lambda}\left(x^{*}\right)-u_{\lambda}(y)\right)-G\left(u\left(x^{*}\right)-u_{\lambda}(y)\right)}{\left|x^{*}-y^{\lambda}\right|^{n+s p}} d y \\
& +C_{n, s, p} \int_{\Sigma_{\lambda}} \frac{G\left(u_{\lambda}\left(x^{*}\right)-u(y)\right)-G\left(u\left(x^{*}\right)-u(y)\right)}{\left|x^{*}-y^{\lambda}\right|^{n+s p}} d y .
\end{aligned}
$$

Clearly,

$$
\frac{1}{\left|x^{*}-y\right|^{n+s p}}-\frac{1}{\left|x^{*}-y^{\lambda}\right|^{n+s p}}>0 .
$$

By the mean value theorem, we also have

$$
\begin{aligned}
G\left(u_{\lambda}\left(x^{*}\right)-u_{\lambda}(y)\right)-G\left(u\left(x^{*}\right)-u(y)\right) & =G^{\prime}\left(\eta_{1}\right)\left[U_{\lambda}\left(x^{*}\right)-U_{\lambda}(y)\right] \leq 0, \\
G\left(u_{\lambda}\left(x^{*}\right)-u_{\lambda}(y)\right)-G\left(u\left(x^{*}\right)-u_{\lambda}(y)\right) & =G^{\prime}\left(\eta_{2}\right) U_{\lambda}\left(x^{*}\right) \leq 0, \\
G\left(u_{\lambda}\left(x^{*}\right)-u(y)\right)-G\left(u\left(x^{*}\right)-u(y)\right) & =G^{\prime}\left(\eta_{3}\right) U_{\lambda}\left(x^{*}\right) \leq 0,
\end{aligned}
$$

where

$$
\begin{gathered}
u_{\lambda}\left(x^{*}\right)-u_{\lambda}(y) \leq \eta_{1} \leq u\left(x^{*}\right)-u(y), \\
u_{\lambda}\left(x^{*}\right)-u_{\lambda}(y) \leq \eta_{2} \leq u\left(x^{*}\right)-u_{\lambda}(y), \\
u_{\lambda}\left(x^{*}\right)-u(y) \leq \eta_{3} \leq u\left(x^{*}\right)-u(y) .
\end{gathered}
$$


Combining (2.1), 2.2) and (2.3), we deduce

$$
(-\Delta)_{p}^{s} u_{\lambda}\left(x^{*}\right)-(-\Delta)_{p}^{s} u\left(x^{*}\right) \leq C_{n, s, p} \int_{\Sigma_{\lambda}} \frac{G\left(u_{\lambda}\left(x^{*}\right)-u(y)\right)-G\left(u\left(x^{*}\right)-u(y)\right)}{\left|x^{*}-y^{\lambda}\right|^{n+s p}} d y .
$$

Then we may apply the Lemma 2.3 to obtain

$$
(-\Delta)_{p}^{s} u_{\lambda}\left(x^{*}\right)-(-\Delta)_{p}^{s} u\left(x^{*}\right) \leq C U_{\lambda}\left(x^{*}\right) \int_{\Sigma_{\lambda}} \frac{\left|u\left(x^{*}\right)-u(y)\right|^{p-2}}{\left|x^{*}-y^{\lambda}\right|^{n+s p}} d y .
$$

From the decay property of $u$, we have

$$
\frac{C_{1}}{|y|^{\alpha}}<u(y)<\frac{C_{2}}{|y|^{\alpha}} \quad \text { for all } y \in \mathbb{R}^{n} \backslash B_{|\bar{\lambda}|},
$$

where constants $C_{1}, C_{2}>0$ depend only on $\bar{\lambda}$. We denote $M=\left(\frac{2 C_{2}}{C_{1}}\right)^{1 / \alpha}$. Then for any $x, y \in \mathbb{R}^{n}$ such that $|y|>M|x|$ and $|x| \geq|\bar{\lambda}|$, we deduce from (2.5)

$$
u(y)<\frac{C_{2}}{|y|^{\alpha}}<\frac{C_{1}}{2|x|^{\alpha}}<\frac{u(x)}{2} .
$$

Next, we choose $z=\left(-(M+1)\left|x^{*}\right|, 0, \ldots, 0\right)$, then $|z|=(M+1)\left|x^{*}\right|$ and $B_{\left|x^{*}\right|}(z) \subset \Sigma_{\lambda}$. Moreover, $u(y)<\frac{1}{2} u\left(x^{*}\right)$ for all $y \in B_{\left|x^{*}\right|}(z)$. Therefore,

$$
\begin{aligned}
\int_{\Sigma_{\lambda}} \frac{\left|u\left(x^{*}\right)-u(y)\right|^{p-2}}{\left|x^{*}-y^{\lambda}\right|^{n+s p}} d y & \geq \frac{u^{p-2}\left(x^{*}\right)}{2^{p-2}} \int_{B_{\left|x^{*}\right|}(z)} \frac{d y}{\left|x^{*}-y^{\lambda}\right|^{n+s p}} \\
& \geq \frac{u^{p-2}\left(x^{*}\right)}{2^{p-2}} \int_{B_{\left|x^{*}\right|}(z)} \frac{d y}{\left[(M+3)\left|x^{*}\right|\right]^{n+s p}} \\
& \geq C \frac{u^{p-2}\left(x^{*}\right)}{\left|x^{*}\right| s p}
\end{aligned}
$$

where we have used the fact

$$
\left|x^{*}-y^{\lambda}\right| \leq\left|x^{*}\right|+\left|-z^{\lambda}\right|+\left|z^{\lambda}-y^{\lambda}\right| \leq\left|x^{*}\right|+|z|+|z-y| \leq(M+3)\left|x^{*}\right|
$$

for all $y \in B_{\left|x^{*}\right|}(z)$. Collecting (2.4), (2.5) and (2.6), we obtain

$$
(-\Delta)_{p}^{s} u_{\lambda}\left(x^{*}\right)-(-\Delta)_{p}^{s} u\left(x^{*}\right) \leq C U_{\lambda}\left(x^{*}\right) \frac{u^{p-2}\left(x^{*}\right)}{\left|x^{*}\right|^{s p}} \leq \frac{C U_{\lambda}\left(x^{*}\right)}{\left|x^{*}\right|^{\alpha(p-2)+s p}} .
$$

This proves the lemma.

3. Symmetry of solutions to fractional systems in the unit ball

We prove Theorem 1.1 in this section. A usual application of the moving plane method in the unit ball consists of two steps: showing that the plane can move from $\lambda=-1$ a little bit to the right, then moving the plane to its limiting position. However, since the plane can move from $\lambda=-\infty$ to $\lambda=-1$ already, we find that Step 1 is unnecessary. Therefore, we go directly to Step 2. Hence, our proof is simpler and requires fewer technical assumptions on $f$ and $g$ comparing to 8,9$]$. 
Proof of Theorem 1.1. Clearly,

$$
U_{\lambda} \geq 0, \quad V_{\lambda} \geq 0 \quad \text { in } \Sigma_{\lambda}
$$

for all $\lambda \leq-1$. Therefore, we may define

$$
\lambda_{0}=\sup \left\{\lambda \leq 0 \mid U_{\mu} \geq 0, V_{\mu} \geq 0 \text { in } \Sigma_{\mu} \text { for all } \mu \leq \lambda\right\}
$$

We claim that

$$
\lambda_{0}=0
$$

By contradiction, suppose $-1 \leq \lambda_{0}<0$. Since $U_{\lambda}$ and $V_{\lambda}$ depend on $\lambda$ continuously, we have

$$
U_{\lambda_{0}} \geq 0, \quad V_{\lambda_{0}} \geq 0 \quad \text { in } \Sigma_{\lambda_{0}} .
$$

If $-1<\lambda_{0}<0$, we must have

$$
U_{\lambda_{0}}>0, \quad V_{\lambda_{0}}>0 \quad \text { in } \Sigma_{\lambda_{0}} \cap \bar{B}_{1} .
$$

To prove (3.2), we may assume in contrary that $U_{\lambda_{0}}\left(x^{*}\right)=0$ for some $x^{*} \in \Sigma_{\lambda_{0}} \cap \bar{B}_{1}$. Then

$$
\begin{aligned}
(-\Delta)_{p}^{s} u_{\lambda_{0}}\left(x^{*}\right)-(-\Delta)_{p}^{s} u\left(x^{*}\right) & =f\left(u_{\lambda_{0}}\left(x^{*}\right), v_{\lambda_{0}}\left(x^{*}\right)\right)-f\left(u\left(x^{*}\right), v\left(x^{*}\right)\right) \\
& =f\left(u\left(x^{*}\right), v_{\lambda_{0}}\left(x^{*}\right)\right)-f\left(u\left(x^{*}\right), v\left(x^{*}\right)\right) \geq 0 .
\end{aligned}
$$

Moreover, since

$$
U_{\lambda_{0}}\left(x^{*}\right)=\min _{\Sigma_{\lambda_{0}}} U_{\lambda_{0}}=0,
$$

the identity 2.1 and 2.3 indicate

$$
\begin{aligned}
& (-\Delta)_{p}^{s} u_{\lambda_{0}}\left(x^{*}\right)-(-\Delta)_{p}^{s} u\left(x^{*}\right) \\
& =C_{n, s, p} \mathrm{PV} \int_{\Sigma_{\lambda_{0}}}\left(\frac{1}{\left|x^{*}-y\right|^{n+s p}}-\frac{1}{\left|x^{*}-y^{\lambda_{0}}\right|^{n+s p}}\right) \\
& \times\left[G\left(u_{\lambda_{0}}\left(x^{*}\right)-u_{\lambda_{0}}(y)\right)-G\left(u\left(x^{*}\right)-u(y)\right)\right] d y \\
& <0 \text {, }
\end{aligned}
$$

where in the last estimation we have used the strict monotonicity of $G$ and the fact

$$
\left[u_{\lambda_{0}}\left(x^{*}\right)-u_{\lambda_{0}}(y)\right]-\left[u\left(x^{*}\right)-u(y)\right]=-u_{\lambda_{0}}(y)<0 \quad \text { for } y \in B_{1}\left(0^{\lambda_{0}}\right) \backslash B_{1} \text {. }
$$

Since (3.4) contradicts (3.3), we must have $U_{\lambda_{0}}>0$ in $\Sigma_{\lambda_{0}} \cap \bar{B}_{1}$. The positivity of $V_{\lambda_{0}}$ in $\Sigma_{\lambda_{0}} \cap \bar{B}_{1}$ can be obtained in the same way. Hence $(3.2)$ is proved. 
By the definition of $\lambda_{0}$, there exists a sequence $\lambda_{k} \searrow \lambda_{0}$ and $x^{k} \in \Sigma_{\lambda_{k}} \cap B_{1}$ such that $\lambda_{k}<0$ and

$$
U_{\lambda_{k}}\left(x^{k}\right)=\min _{\Sigma_{\lambda_{k}}} U_{\lambda_{k}}<0 \quad \text { or } \quad V_{\lambda_{k}}\left(x^{k}\right)=\min _{\Sigma_{\lambda_{k}}} V_{\lambda_{k}}<0 .
$$

Without loss of generality, by passing to a subsequence, we may assume

$$
U_{\lambda_{k}}\left(x^{k}\right)=\min _{\Sigma_{\lambda_{k}}} U_{\lambda_{k}}<0 \quad \text { and } \quad x^{k} \rightarrow x^{0} \in \bar{\Sigma}_{\lambda_{0}} \cap \bar{B}_{1} .
$$

Moreover, since $x^{k} \in \Sigma_{\lambda_{k}} \cap B_{1}$ is the interior minimum point, we have

$$
\nabla U_{\lambda_{k}}\left(x^{k}\right)=0
$$

From the continuity of $U_{\lambda}(x)$ and its derivative with respect to both $x$ and $\lambda$, we deduce

$$
U_{\lambda_{0}}\left(x^{0}\right) \leq 0 \quad \text { and } \quad \nabla U_{\lambda_{0}}\left(x^{0}\right)=0 .
$$

If $\lambda_{0}=-1$, then $x^{0}=(-1,0, \ldots, 0) \in T_{\lambda_{0}}$. If $-1<\lambda_{0}<0$, then we also have $x^{0} \in T_{\lambda_{0}}$ thanks to 3.2 . Define $\delta_{k}$ as in Lemma 2.1. It follows that

$$
\frac{U_{\lambda_{k}}\left(x^{k}\right)}{\delta_{k}} \rightarrow-\frac{\partial U_{\lambda_{0}}\left(x^{0}\right)}{\partial x_{1}}=0 \quad \text { as } k \rightarrow \infty .
$$

We also have

$$
\frac{V_{\lambda_{k}}\left(x^{k}\right)}{\delta_{k}} \rightarrow-\frac{\partial V_{\lambda_{0}}\left(x^{0}\right)}{\partial x_{1}} \geq 0 \quad \text { as } k \rightarrow \infty .
$$

On the other hand, we have the formulae

$$
\begin{aligned}
& (-\Delta)_{p}^{s} u_{\lambda}(x)-(-\Delta)_{p}^{s} u(x) \\
= & f\left(u_{\lambda}(x), v_{\lambda}(x)\right)-f(u(x), v(x)) \\
= & {\left[f\left(u_{\lambda}(x), v(x)\right)-f(u(x), v(x))\right]+\left[f\left(u_{\lambda}(x), v_{\lambda}(x)\right)-f\left(u_{\lambda}(x), v(x)\right)\right] } \\
\geq & F_{\lambda}(x) U_{\lambda}(x)+G_{\lambda}(x) V_{\lambda}(x)^{-},
\end{aligned}
$$

where

$$
\begin{aligned}
& F_{\lambda}(x)= \begin{cases}\frac{f\left(u_{\lambda}(x), v(x)\right)-f(u(x), v(x))}{u_{\lambda}(x)-u(x)} & \text { if } u_{\lambda}(x) \neq u(x), \\
0 & \text { if } u_{\lambda}(x)=u(x),\end{cases} \\
& G_{\lambda}(x)= \begin{cases}\frac{f\left(u_{\lambda}(x), v_{\lambda}(x)\right)-f\left(u_{\lambda}(x), v(x)\right)}{v_{\lambda}(x)-v(x)} & \text { if } v_{\lambda}(x)<v(x), \\
0 & \text { if } v_{\lambda}(x) \geq v(x) .\end{cases}
\end{aligned}
$$

Notice that $u_{\lambda_{k}}\left(x^{k}\right)<u\left(x^{k}\right) \leq \max _{\bar{B}_{1}} u$ and $v_{\lambda_{k}}\left(x^{k}\right), v\left(x^{k}\right) \leq \max _{\bar{B}_{1}} v$. Then we may use the local Lipschitz continuity of $f$ to deduce that $F_{\lambda_{k}}\left(x^{k}\right) \leq C$ and $G_{\lambda_{k}}\left(x^{k}\right) \leq C$. Therefore, by combining (3.5), (3.6) and (3.7), we obtain

$$
\frac{1}{\delta_{k}}\left[(-\Delta)_{p}^{s} u_{\lambda_{k}}\left(x^{k}\right)-(-\Delta)_{p}^{s} u\left(x^{k}\right)\right] \geq C\left(\frac{U_{\lambda_{k}}\left(x^{k}\right)}{\delta_{k}}+\frac{V_{\lambda_{k}}\left(x^{k}\right)^{-}}{\delta_{k}}\right) \rightarrow 0 .
$$


However, this contradicts Lemma 2.1. Hence (3.1) is proved.

Since the direction $x_{1}$ can be chosen arbitrarily, we deduce that $u$ and $v$ are radially symmetric and monotone decreasing about the origin. This completes the proof of Theorem 1.1 .

\section{Symmetry of solutions to fractional systems in $\mathbb{R}^{n}$}

We assume that all assumptions of Theorem 1.2 are satisfied.

Lemma 4.1 (Decay at infinity principle). Let $\bar{\lambda}<0$. There exists $R_{0}>0$ such that for all $\lambda \leq \bar{\lambda}$, we have the following claims:

(i) If $x^{*} \in \Sigma_{\lambda} \backslash B_{R_{0}}$ and $U_{\lambda}\left(x^{*}\right)=\min _{\Sigma_{\lambda}} U_{\lambda}<0$, then

$$
V_{\lambda}\left(x^{*}\right)<2 U_{\lambda}\left(x^{*}\right)
$$

(ii) If $x^{*} \in \Sigma_{\lambda} \backslash B_{R_{0}}$ and $V_{\lambda}\left(x^{*}\right)=\min _{\Sigma_{\lambda}} V_{\lambda}<0$, then

$$
U_{\lambda}\left(x^{*}\right)<2 V_{\lambda}\left(x^{*}\right)
$$

Proof. Let us assume

$$
U_{\lambda}\left(x^{*}\right)=\min _{\Sigma_{\lambda}} U_{\lambda}<0
$$

for some $x^{*} \in \Sigma_{\lambda}$. Clearly, if there exists some $R_{0}$ independent of $\lambda$ such that $\left|x^{*}\right|<R_{0}$, then (i) is true. Therefore, in what follows, we may assume that $\left|x^{*}\right|$ is sufficiently large. We have

$$
\begin{aligned}
& (-\Delta)_{p}^{s} u_{\lambda}(x)-(-\Delta)_{p}^{s} u(x) \\
= & f\left(u_{\lambda}(x), v_{\lambda}(x)\right)-f(u(x), v(x)) \\
= & {\left[f\left(u_{\lambda}(x), v(x)\right)-f(u(x), v(x))\right]+\left[f\left(u_{\lambda}(x), v_{\lambda}(x)\right)-f\left(u_{\lambda}(x), v(x)\right)\right] } \\
= & f_{u}^{\prime}\left(\xi_{\lambda}(x), v(x)\right) U_{\lambda}(x)+f_{v}^{\prime}\left(u_{\lambda}(x), \zeta_{\lambda}(x)\right) V_{\lambda}(x),
\end{aligned}
$$

where $\xi_{\lambda}(x)$ is between $u_{\lambda}(x)$ and $u(x) ; \zeta_{\lambda}(x)$ is between $v_{\lambda}(x)$ and $v(x)$. Combining (4.1) and Lemma 2.4, we obtain

$$
\left(\frac{C}{\left|x^{*}\right|^{\alpha(p-2)+s p}}-f_{u}^{\prime}\left(\xi_{\lambda}\left(x^{*}\right), v\left(x^{*}\right)\right)\right) U_{\lambda}\left(x^{*}\right) \geq f_{v}^{\prime}\left(u_{\lambda}\left(x^{*}\right), \zeta_{\lambda}\left(x^{*}\right)\right) V_{\lambda}\left(x^{*}\right) .
$$

If $\left|x^{*}\right|$ is sufficiently large, by the assumptions (ii) and (iii), we have

$$
f_{u}^{\prime}\left(\xi_{\lambda}\left(x^{*}\right), v\left(x^{*}\right)\right) \leq 0
$$

or

$$
f_{u}^{\prime}\left(\xi_{\lambda}\left(x^{*}\right), v\left(x^{*}\right)\right)=o(1) \xi_{\lambda}\left(x^{*}\right)^{a_{1}} v\left(x^{*}\right)^{b_{1}}=\frac{o(1)}{\left|x^{*}\right|^{\alpha a_{1}+\beta b_{1}}}
$$


where $o(1) \rightarrow 0$ as $\left|x^{*}\right| \rightarrow \infty$. Hence for $\left|x^{*}\right|$ sufficiently large, the left-hand side of (4.2) is negative. This implies $V_{\lambda}\left(x^{*}\right)<0$ and $\zeta_{\lambda}\left(x^{*}\right)<v\left(x^{*}\right)$, which is small. Then we have

$$
f_{v}^{\prime}\left(u_{\lambda}\left(x^{*}\right), \zeta_{\lambda}\left(x^{*}\right)\right)=o(1) \xi_{\lambda}\left(x^{*}\right)^{a_{2}} v\left(x^{*}\right)^{b_{2}}=\frac{o(1)}{\left|x^{*}\right|^{\alpha a_{2}+\beta b_{2}}} .
$$

Therefore, from (4.2), we conclude that

$$
V_{\lambda}\left(x^{*}\right)<2 U_{\lambda}\left(x^{*}\right)
$$

if $\left|x^{*}\right| \geq R_{0}$ for some $R_{0}>0$ sufficiently large. Hence, (i) holds for such an $R_{0}$.

In the same way, we can choose $R_{0}$, which is larger if necessary, such that (ii) also holds.

Proof of Theorem 1.2. The asymptotic properties of $u$ and $v$ indicate that, if $U_{\lambda}$ or $V_{\lambda}$ is negative somewhere in $\Sigma_{\lambda}$, then its negative minima are attained in the interior of $\Sigma_{\lambda}$. We carry out the method of moving planes along the $x_{1}$ direction in two steps.

Step 1: Move the plane along $x_{1}$ direction from near $-\infty$. In this step, we show that for $\lambda$ sufficiently negative,

$$
U_{\lambda} \geq 0, \quad V_{\lambda} \geq 0 \quad \text { in } \Sigma_{\lambda}
$$

Indeed, we may choose $\lambda \leq-R_{0}$, where $R_{0}$ is defined in Lemma 4.1 with $\bar{\lambda}=-1$. If (4.3) does not hold for such a $\lambda$, then there exists $x^{*} \in \Sigma_{\lambda}$ such that $U_{\lambda}\left(x^{*}\right)=\min _{\Sigma_{\lambda}} U_{\lambda}<0$ or $V_{\lambda}\left(x^{*}\right)=\min _{\Sigma_{\lambda}} V_{\lambda}<0$. Without loss of generality, we may assume

$$
U_{\lambda}\left(x^{*}\right)=\min _{\Sigma_{\lambda}} U_{\lambda}<0
$$

Since $\left|x^{*}\right|>|\lambda| \geq R_{0}$, we may use Lemma 4.1 to deduce

$$
V_{\lambda}\left(x^{*}\right)<2 U_{\lambda}\left(x^{*}\right)<0 .
$$

Hence, there exists $y^{*} \in \Sigma_{\lambda}$ such that

$$
V_{\lambda}\left(y^{*}\right)=\min _{\Sigma_{\lambda}} V_{\lambda}<0
$$

Using Lemma 4.1 again, we obtain

$$
U_{\lambda}\left(y^{*}\right)<2 V_{\lambda}\left(y^{*}\right) \text {. }
$$

Combining 4.4 and 4.5, we get

$$
V_{\lambda}\left(x^{*}\right)<2 U_{\lambda}\left(x^{*}\right) \leq 2 U_{\lambda}\left(y^{*}\right)<4 V_{\lambda}\left(y^{*}\right) \leq 4 V_{\lambda}\left(x^{*}\right),
$$


which implies $V_{\lambda}\left(x^{*}\right)>0$. However, this contradicts (4.4). Hence (4.3) holds for all $\lambda \leq-R_{0}$.

Step 2: Move the plane to the limiting position. Step 1 provides a starting point, from which we can now move the plane $T_{\lambda}$ to the right as long as (4.3) holds to its limiting position. Define

$$
\lambda_{0}=\sup \left\{\lambda \leq 0 \mid U_{\mu} \geq 0, V_{\mu} \geq 0 \text { in } \Sigma_{\mu} \text { for all } \mu \leq \lambda\right\}
$$

In what follows, we assume $\lambda_{0}<0$. Since $U_{\lambda}$ and $V_{\lambda}$ depend on $\lambda$ continuously, we already have

$$
U_{\lambda_{0}} \geq 0, \quad V_{\lambda_{0}} \geq 0 \quad \text { in } \Sigma_{\lambda_{0}}
$$

There are two possible cases:

Case 1: $U_{\lambda_{0}}>0$ and $V_{\lambda_{0}}>0$ in $\Sigma_{\lambda_{0}}$. By the definition of $\lambda_{0}$, there exists a sequence $\lambda_{k} \searrow \lambda_{0}$ and $x^{k} \in \Sigma_{\lambda_{k}}$ such that $\lambda_{k}<\lambda_{0} / 2$ and

$$
U_{\lambda_{k}}\left(x^{k}\right)=\min _{\Sigma_{\lambda_{k}}} U_{\lambda_{k}}<0 \quad \text { or } \quad V_{\lambda_{k}}\left(x^{k}\right)=\min _{\Sigma_{\lambda_{k}}} V_{\lambda_{k}}<0 .
$$

Up to a subsequence, we may assume

$$
U_{\lambda_{k}}\left(x^{k}\right)=\min _{\Sigma_{\lambda_{k}}} U_{\lambda_{k}}<0
$$

Let $R_{0}$ be defined as in Lemma 4.1 with $\bar{\lambda}=\lambda_{0} / 2$. There are two possibilities.

Possibility (i): Up to a subsequence, $\left|x^{k}\right|<R_{0}$. We may assume that $x^{k} \rightarrow x^{0} \in \bar{\Sigma}_{\lambda_{0}}$. Moreover, since $x^{k} \in \Sigma_{\lambda_{k}}$ is the interior minimum point, we have

$$
\nabla U_{\lambda_{k}}\left(x^{k}\right)=0
$$

From the continuity of $U_{\lambda}(x)$ and its derivative with respect to both $x$ and $\lambda$, we have

$$
U_{\lambda_{0}}\left(x^{0}\right) \leq 0 \quad \text { and } \quad \nabla U_{\lambda_{0}}\left(x^{0}\right)=0
$$

Hence $x^{0} \in T_{\lambda_{0}}$. It follows that

$$
\frac{U_{\lambda_{k}}\left(x^{k}\right)}{\delta_{k}} \rightarrow-\frac{\partial U_{\lambda_{0}}\left(x^{0}\right)}{\partial x_{1}}=0 \quad \text { as } k \rightarrow \infty .
$$

We also have

$$
\frac{V_{\lambda_{k}}\left(x^{k}\right)}{\delta_{k}} \rightarrow-\frac{\partial V_{\lambda_{0}}\left(x^{0}\right)}{\partial x_{1}} \geq 0 \quad \text { as } k \rightarrow \infty .
$$


On the other hand, we may use (4.1) to obtain

$$
\begin{aligned}
& \frac{1}{\delta_{k}}\left[(-\Delta)_{p}^{s} u_{\lambda_{k}}\left(x^{k}\right)-(-\Delta)_{p}^{s} u\left(x^{k}\right)\right] \\
= & f_{u}^{\prime}\left(\xi_{\lambda_{k}}\left(x^{k}\right), v\left(x^{k}\right)\right) \frac{U_{\lambda_{k}}\left(x^{k}\right)}{\delta_{k}}+f_{v}^{\prime}\left(u_{\lambda_{k}}\left(x^{k}\right), \zeta_{\lambda_{k}}\left(x^{k}\right)\right) \frac{V_{\lambda_{k}}\left(x^{k}\right)}{\delta_{k}} \\
\geq & \left(\max _{\Omega} f_{u}^{\prime}\right) \frac{U_{\lambda_{k}}\left(x^{k}\right)}{\delta_{k}}+\left(\max _{\Omega} f_{v}^{\prime}\right) \frac{V_{\lambda_{k}}\left(x^{k}\right)^{-}}{\delta_{k}}
\end{aligned}
$$

for sufficiently large $k$, where

$$
\Omega=\left[\min _{\bar{B}_{1}\left(x^{0}\right)} u, \underset{\max _{1}\left(x^{0}\right)}{ } u\right] \times\left[\min _{B_{1}\left(x^{0}\right)} v, \underset{B_{1}\left(x^{0}\right)}{\max } v\right] .
$$

Combining (4.6) and (4.7) with the above estimation, we obtain

$$
\liminf _{\delta_{k} \rightarrow 0} \frac{1}{\delta_{k}}\left[(-\Delta)_{p}^{s} u_{\lambda_{k}}\left(x^{k}\right)-(-\Delta)_{p}^{s} u\left(x^{k}\right)\right] \geq 0 .
$$

However, this contradicts Lemma 2.1.

Possibility (ii): $\left|x^{k}\right| \geq R_{0}$ for all $k \geq k_{0}$. By Lemma 4.1. $V_{\lambda_{k}}\left(x^{k}\right)<2 U_{\lambda_{k}}\left(x^{k}\right)<0$. Hence, there exits $y^{k} \in \Sigma_{\lambda_{k}}$ such that

$$
V_{\lambda_{k}}\left(y^{k}\right)=\min _{\Sigma_{\lambda_{k}}} V_{\lambda_{k}}<0
$$

If $\left|y^{k}\right| \geq R_{0}$, then we get a contradiction as in Step 1 .

Thus, we must have $\left|y^{k}\right|<R_{0}$ for all $k \geq k_{0}$. Repeating the arguments in Possibility (i) for $V_{\lambda_{k}}$ and $y^{k}$, we can derive a contradiction with Lemma 2.1. Therefore, Case 1 cannot happen.

Case 2: $U_{\lambda_{0}}\left(x^{*}\right)=0$ or $V_{\lambda_{0}}\left(x^{*}\right)=0$ for some $x^{*} \in \Sigma_{\lambda_{0}}$. We may assume $U_{\lambda_{0}}\left(x^{*}\right)=0$. From 4.1), we have

$$
(-\Delta)_{p}^{s} u_{\lambda_{0}}\left(x^{*}\right)-(-\Delta)_{p}^{s} u\left(x^{*}\right)=f_{v}^{\prime}\left(u_{\lambda_{0}}\left(x^{*}\right), \zeta_{\lambda_{0}}\left(x^{*}\right)\right) V_{\lambda_{0}}\left(x^{*}\right) \geq 0 .
$$

Moreover, since

$$
U_{\lambda_{0}}\left(x^{*}\right)=\min _{\Sigma_{\lambda_{0}}} U_{\lambda_{0}}=0,
$$

the identity 2.1 and 2.3 imply

$$
\begin{aligned}
&(-\Delta)_{p}^{s} u_{\lambda_{0}}\left(x^{*}\right)-(-\Delta)_{p}^{s} u\left(x^{*}\right) \\
&=C_{n, s, p} \mathrm{PV} \int_{\Sigma_{\lambda_{0}}}\left(\frac{1}{\left|x^{*}-y\right|^{n+s p}}-\frac{1}{\left|x^{*}-y^{\lambda_{0}}\right|^{n+s p}}\right) \\
& \times\left[G\left(u_{\lambda_{0}}\left(x^{*}\right)-u_{\lambda_{0}}(y)\right)-G\left(u\left(x^{*}\right)-u(y)\right)\right] d y
\end{aligned}
$$

$\leq 0$ 
Due to the strict monotonicity of $G$, 4.8 and (4.9) imply $U_{\lambda_{0}} \equiv 0$ in $\Sigma_{\lambda_{0}}$. Then using (4.1) again, we have

$$
0=(-\Delta)_{p}^{s} u_{\lambda_{0}}(x)-(-\Delta)_{p}^{s} u(x)=f_{v}^{\prime}\left(u_{\lambda_{0}}(x), \zeta_{\lambda_{0}}(x)\right) V_{\lambda_{0}}(x) \geq 0 .
$$

Since $f_{v}^{\prime}>0$, we must have $V_{\lambda_{0}} \equiv 0$ in $\Sigma_{\lambda_{0}}$. In summary, if $\lambda_{0}<0$, then $u$ and $v$ are symmetric and monotone decreasing about plane $T_{\lambda_{0}}$.

It remains to consider the case $\lambda_{0}=0$. In this case, $U_{0} \geq 0, V_{0} \geq 0$ in $\Sigma_{0}$. By moving the plane along $x_{1}$ direction from near $+\infty$, we also deduce that either $u$ and $v$ are symmetric and monotone decreasing about a plane $T_{\lambda_{1}}$ for some $\lambda_{1}>0$ or $U_{0} \leq 0$, $V_{0} \leq 0$ in $\Sigma_{0}$. In the latter case, $u$ and $v$ must be symmetric and monotone decreasing about plane $T_{0}$.

We may repeat the above arguments for any direction to conclude that $u$ and $v$ are radially symmetric and monotone decreasing about some point $x^{0} \in \mathbb{R}^{n}$. This completes the proof of Theorem 1.2 .

Proof of Theorem 1.4. We claim that Lemma 4.1 still holds for system (1.4) under assumptions of Theorem 1.4 .

Indeed, we may proceed as in the proof of Lemma 4.1. Instead of (4.1) and (4.2), we now have

$$
\begin{aligned}
& (-\Delta)_{p}^{s} u_{\lambda}\left(x^{*}\right)-(-\Delta)_{p}^{s} u\left(x^{*}\right) \\
= & {\left[u_{\lambda}\left(x^{*}\right)^{a}-u\left(x^{*}\right)^{a}\right] v\left(x^{*}\right)^{b}+\left[v_{\lambda}\left(x^{*}\right)^{b}-v\left(x^{*}\right)^{b}\right] u_{\lambda}\left(x^{*}\right)^{a} } \\
\geq & {\left[u_{\lambda}\left(x^{*}\right)^{a}-u\left(x^{*}\right)^{a}\right] v\left(x^{*}\right)^{b}+\left[v_{\lambda}\left(x^{*}\right)^{b}-v\left(x^{*}\right)^{b}\right]^{-} u_{\lambda}\left(x^{*}\right)^{a} . }
\end{aligned}
$$

If $a \leq 0$, then $\left[u_{\lambda}\left(x^{*}\right)^{a}-u\left(x^{*}\right)^{a}\right] v\left(x^{*}\right)^{b} \geq 0$. If $a>0$, then

$$
\begin{aligned}
{\left[u_{\lambda}\left(x^{*}\right)^{a}-u\left(x^{*}\right)^{a}\right] v\left(x^{*}\right)^{b} } & \geq C\left[u_{\lambda}\left(x^{*}\right)^{a}-u\left(x^{*}\right)^{a}\right] u\left(x^{*}\right)\left|x^{*}\right|^{\alpha-\beta b} \\
& \geq C\left[u_{\lambda}\left(x^{*}\right)^{a+1}-u\left(x^{*}\right)^{a+1}\right]\left|x^{*}\right|^{\alpha-\beta b} \\
& =C U_{\lambda}\left(x^{*}\right) \xi_{\lambda}\left(x^{*}\right)^{a}\left|x^{*}\right|^{\alpha-\beta b} \\
& \geq C U_{\lambda}\left(x^{*}\right) u\left(x^{*}\right)^{a}\left|x^{*}\right|^{\alpha-\beta b} \\
& \geq \frac{C U_{\lambda}\left(x^{*}\right)}{\left|x^{*}\right|^{\alpha(a-1)+\beta b}} .
\end{aligned}
$$

Hence for all $a \in \mathbb{R}$, we have

$$
\left[u_{\lambda}\left(x^{*}\right)^{a}-u\left(x^{*}\right)^{a}\right] v\left(x^{*}\right)^{b} \geq \frac{C a^{+} U_{\lambda}\left(x^{*}\right)}{\left|x^{*}\right|^{\alpha(a-1)+\beta b}} .
$$

Similarly, since $b>0$,

$$
\left[v_{\lambda}\left(x^{*}\right)^{b}-v\left(x^{*}\right)^{b}\right]^{-} u_{\lambda}\left(x^{*}\right)^{a} \geq \frac{C V_{\lambda}\left(x^{*}\right)^{-}}{\left|x^{*}\right|^{\alpha a+\beta(b-1)}} .
$$


Substituting 4.11 and 4.12 into 4.10 and using Lemma 2.4, we obtain

$$
C\left(\frac{1}{\left|x^{*}\right|^{\alpha(p-2)+s p}}-\frac{a^{+}}{\left|x^{*}\right|^{\alpha(a-1)+\beta b}}\right) U_{\lambda}\left(x^{*}\right) \geq \frac{C V_{\lambda}\left(x^{*}\right)^{-}}{\left|x^{*}\right|^{\alpha a+\beta(b-1)}} .
$$

From this and assumptions (i), (ii), we deduce that there exists $R_{0}$ sufficiently large such that

$$
V_{\lambda}\left(x^{*}\right)<2 U_{\lambda}\left(x^{*}\right)
$$

if $\left|x^{*}\right| \geq R_{0}$. This proves Lemma 4.1 .

The remainder of the proof is similar to that of Theorem 1.2 and hence will be omitted.

\section{References}

[1] D. Applebaum, Lévy Processes and Stochastic Calculus, Second edition, Cambridge Studies in Advanced Mathematics 116, Cambridge University Press, Cambridge, 2009.

[2] J. Bertoin, Lévy Processes, Cambridge Tracts in Mathematics 121, Cambridge University Press, Cambridge, 1996.

[3] C. Bjorland, L. Caffarelli and A. Figalli, Non-local gradient dependent operators, Adv. Math. 230 (2012), no. 4-6, 1859-1894.

[4] _ Nonlocal tug-of-war and the infinity fractional Laplacian, Comm. Pure Appl. Math. 65 (2012), no. 3, 337-380.

[5] J.-P. Bouchaud and A. Georges, Anomalous diffusion in disordered media: Statistical mechanisms, models and physical applications, Phys. Rep. 195 (1990), no. 4-5, 127293.

[6] L. Caffarelli and L. Silvestre, An extension problem related to the fractional Laplacian, Comm. Partial Differential Equations 32 (2007), no. 7-9, 1245-1260.

[7] L. A. Caffarelli and A. Vasseur, Drift diffusion equations with fractional diffusion and the quasi-geostrophic equation, Ann. of Math. (2) 171 (2010), no. 3, 1903-1930.

[8] L. Cao, X. Wang and Z. Dai, Radial symmetry and monotonicity of solutions to a system involving fractional p-Laplacian in a ball, Adv. Math. Phys. 2018 (2018), Art. ID 1565731, 6 pp.

[9] W. Chen and C. Li, Maximum principles for the fractional p-Laplacian and symmetry of solutions, Adv. Math. 335 (2018), 735-758. 
[10] W. Chen, C. Li and Y. Li, A direct method of moving planes for the fractional Laplacian, Adv. Math. 308 (2017), 404-437.

[11] W. Chen, C. Li and B. Ou, Classification of solutions for an integral equation, Comm. Pure Appl. Math. 59 (2006), no. 3, 330-343.

[12] Y. Chen and B. Liu, Symmetry and non-existence of positive solutions for fractional p-Laplacian systems, Nonlinear Anal. 183 (2019), 303-322.

[13] C. Cheng, Z. Lü and Y. Lü, A direct method of moving planes for the system of the fractional Laplacian, Pacific J. Math. 290 (2017), no. 2, 301-320.

[14] P. Constantin, Euler equations, Navier-Stokes equations and turbulence, in: Mathematical Foundation of Turbulent Viscous Flows, 1-43, Lecture Notes in Math. 1871, Springer, Berlin, 2006.

[15] P. Felmer and Y. Wang, Radial symmetry of positive solutions to equations involving the fractional Laplacian, Commun. Contemp. Math. 16 (2014), no. 1, 1350023, 24 pp.

[16] S. Jarohs and T. Weth, Symmetry via antisymmetric maximum principles in nonlocal problems of variable order, Ann. Mat. Pura Appl. (4) 195 (2016), no. 1, 273-291.

[17] P. Le, Liouville theorem and classification of positive solutions for a fractional Choquard type equation, Nonlinear Anal. 185 (2019), 123-141.

[18] Liouville theorem for fractional Hénon-Lane-Emden systems on a half space, Proc. Roy. Soc. Edinburgh Sect. A 150 (2020), no. 6, 3060-3073.

[19] _ Symmetry of solutions for a fractional p-Laplacian equation of Choquard type, Internat. J. Math. 31 (2020), no. 4, 2050026, 14 pp.

[20] Y. Li and P. Ma, Symmetry of solutions for a fractional system, Sci. China Math. 60 (2017), no. 10, 1805-1824.

[21] B. Liu and L. Ma, Radial symmetry results for fractional Laplacian systems, Nonlinear Anal. 146 (2016), 120-135.

[22] L. Ma and Z. Zhang, Symmetry of positive solutions for Choquard equations with fractional p-Laplacian, Nonlinear Anal. 182 (2019), 248-262.

[23] P. Ma, Y. Li and J. Zhang, Symmetry and nonexistence of positive solutions for fractional systems, Commun. Pure Appl. Anal. 17 (2018), no. 3, 1053-1070. 
[24] G. Molica Bisci, V. D. Radulescu and R. Servadei, Variational Methods for Nonlocal Fractional Problems, Encyclopedia of Mathematics and its Applications 162, Cambridge University Press, Cambridge, 2016.

[25] A. Quaas and A. Xia, A Liouville type theorem for Lane-Emden systems involving the fractional Laplacian, Nonlinearity 29 (2016), no. 8, 2279-2297.

[26] L. Wu and P. Niu, Symmetry and nonexistence of positive solutions to fractional p-Laplacian equations, Discrete Contin. Dyn. Syst. 39 (2019), no. 3, 1573-1583.

[27] Z. Wu and H. Xu, Symmetry properties in systems of fractional Laplacian equations, Discrete Contin. Dyn. Syst. 39 (2019), no. 3, 1559-1571.

[28] R. Zhuo and Y. Li, Nonexistence and symmetry of solutions for Schrödinger systems involving fractional Laplacian, Discrete Contin. Dyn. Syst. 39 (2019), no. 3, 15951611.

Phuong Le

Faculty of Economic Mathematics, University of Economics and Law, Ho Chi Minh City, Vietnam

and

Vietnam National University, Ho Chi Minh City, Vietnam

E-mail address: phuongl@uel.edu.vn 